\title{
Study on a two-component signal transduction system RimA1A2 that negatively regulates oxytetracycline biosynthesis in Streptomyces rimosus M4018
}

\author{
Hui $\mathrm{Ni}^{1}$, Zhiqiang Xiong ${ }^{2}$, Ali Mohsin ${ }^{1}$, Meijin Guo $^{1 *} \mathbb{C}$, Hrvoje Petkovic $^{3}, \mathrm{Ju}_{\mathrm{Ch}}{ }^{1}$ and Yingping Zhuang ${ }^{{ }^{*}}$
}

\begin{abstract}
Biosynthesis of secondary metabolites in actinobacteria is regulated by complex regulatory mechanisms on responding environmental changes. In this study, we have identified a two-component system (TCS) designated as RimA1A2 in the genome of Streptomyces rimosus M4018, with high homology to the TCS RapA1A2 from Streptomyces coelicolor, known for its positive regulatory function towards actinorhodin (ACT) biosynthesis. Using RT-PCR analysis, we demonstrate that rimA1 encodes response regulator (RR) and rimA2 encoding histidine kinase (HK) from S. rimosus that are co-transcribed as a single-polycistronic mRNA. When S. rimosus was cultivated on standard media, no significant difference in culture growth or morphological properties was observed between the rimA1-disrupted mutant and parent strain M4018. However, the rimA1-disrupted strain displayed significant increase in oxytetracycline (OTC) titer when cultivated in minimal medium (MM) containing glycine as sole nitrogen source, and the transcription of selected genes involved in OTC biosynthesis was increased, supporting the hypothesis that RimA1A2 has a negative regulatory role in OTC biosynthesis via global regulation. We observed an increased resistance of the rimA1-disrupted mutant strain to selected antibiotics. Interestingly, in the rimA 1-disrupted strain, OTC biosynthesis was affected under different environmental stress conditions such as osmotic and oxidative stress. Accordingly, this phenotype was observed in a medium-dependent manner. Considering complexity of regulatory networks in antibiotic-producing organisms, this study demonstrates the importance of cultivation conditions, which is often neglected.
\end{abstract}

Keywords: RimA1A2, Oxytetracycline biosynthesis, Two-component regulatory system, Environmental stress, Streptomyces rimosus, Negative regulation

\section{Introduction}

Streptomyces rimosus is a Gram-positive, aerobic, and filamentous actinobacterium, which produces the medically important broad-spectrum antibiotic oxytetracycline (OTC) (Finlay et al. 1950). S. rimosus is the main commercial OTC producing strain, and the biosynthesis of OTC has been extensively studied (Petkovic et al. 2017). The genome of wild-type S. rimosus ATCC 10970

\footnotetext{
*Correspondence: guo_mj@ecust.edu.cn; ypzhuang@ecust.edu.cn ${ }^{1}$ State Key Laboratory of Bioreactor Engineering, East China University of Science and Technology, P.O. box 329\#, 130 Meilong Rd, Shanghai 200237, People's Republic of China

Full list of author information is available at the end of the article
}

strain was sequenced in our previous study (Pethick et al. 2013).

The pathway-specific regulators and the global regulators play important roles in the regulation of secondary metabolism of antibiotic production. These are capable of repressing or triggering the expression of genes involved in biosynthesis of antibiotic. To date, the Streptomyces antibiotic regulatory proteins (SARPs) are the best-characterized pathway-specific regulators, which possess an OmpR-like winged helix-turn-helix (HTH) DNA-binding motif near the $\mathrm{N}$-terminus and an adjacent bacterial transcriptional activation domain (BTAD) (Wietzorrek and Bibb 1997). As recently demonstrated, an SARP positive regulatory protein OtcR was located in 
OTC biosynthesis gene cluster oxy in S. rimosus. Regulatory protein OtcR directly activates five promoters in $o x y$ cluster (Yin et al. 2015).

When considering global regulators, many of pleiotropic regulatory proteins belong to TCSs, which is the major signal transduction system used by bacteria to sense and respond the environmental stimuli (Hakenbeck and Stock 1996; Bijlsma and Groisman 2003). Naturally, typical TCS contains a histidine kinase (HK) bound on cell membrane that senses specific environmental stimuli and a corresponding response regulator (RR) that mediates the cellular response mainly through the transcriptional regulation of target genes (Mascher et al. 2006). Moreover, TCS has been considered to play an active role in different kinds of bacterial cellular responses, for instance, sporulation, chemotaxis, osmoregulation, antibiotic production, and so on (Ogura and Tanaka 2002; Bijlsma and Groisman 2003; Hutchings et al. 2004). In general, when environmental signals detected by TCS are achieved by autophosphorylation of the histidine kinase at a conserved histidine residue. The phosphorylation of an aspartic acid residue of the response regulator results from the phosphorylated histidine of these histidine kinases. A conformational change is induced by the phosphorylation of the response regulator proteins, which alters their binding properties.

Streptomyces coelicolor is genetically the most studied Streptomycetes strain (Bentley et al. 2002) and bioinformatics analysis of the complete genome sequence of $S$. coelicolor revealed 84 putative histidine kinase genes and 80 putative response regulator genes, and 67 typical TCSs was found out from these HKs and RRs. Although only a limited number of the TCS gene homologues have been studied, it clearly implies that TCSs play important physiological roles in S. coelicolor. So far, only few TCSs have been identified to play their roles in the regulation of antibiotic biosynthesis in S. coelicolor, such as AbsA1A2 (Adamidis et al. 1990; Brian et al. 1996; Ryding et al. 2002; McKenzie and Nodwell 2007), AfsQ1Q2 (Shu et al. 2009; Wang et al. 2013), CseBC (Paget et al. 1999), DraRK (Yu et al. 2012a, b), PhoRP (Sola-Landa et al. 2005; Rodriguez-Garcia et al. 2007; Sola-Landa et al. 2008; SantosBeneit et al. 2009), and RapA1A2 (Lu et al. 2007). For example, positive regulatory role of the RapA1A2 in actinorhodin biosynthesis was observed in S. coelicolor on rich $\mathrm{R} 4 \mathrm{C}$ medium (Lu et al. 2007).

In this study, a TCS RimA1A2 in S. rimosus with high similarity to TCS RapA1A2 in S. coelicolor was identified and characterized. After thorough evaluation of phenotype of the $S$. rimosus rimA1-disrupted mutant strain culture under diverse cultivation conditions, and under influence of different environmental stress conditions, we demonstrate that RimA1A2 plays a significant role in regulation of OTC biosynthesis only under specific environmental stress conditions, which are, however, not comparable to the optimal conditions used for industrial OTC production. Finally, we have also observed the role of RimA1A2 in OTC biosynthesis in relation with the osmotic and oxidative stress response, thus presenting the complex regulatory role of this TCS in S. rimosus.

\section{Methods}

\section{Strains and culture conditions}

Bacterial strains and plasmids used in this work are shown in Table 1. S. rimosus M4018, a type strain, was used as the donor of DNA as well as the parental strain for strain construction. E. coli DH5 $\alpha$ was used for regular cloning. S. rimosus M4018 and its derivatives were grown on wheat bran medium (2.5\% agar, $7.5 \%$ wheat bran) at $30{ }^{\circ} \mathrm{C}$ for spore suspension preparation. Solid minimal medium in which L-asparagine (Asn) was replaced by $50 \mathrm{mM}$ L-glycine (Gly), as the sole nitrogen source was used for OTC production determination, growth curves, and RNA preparation. The mycelium of $S$. rimosus was grown on Tryptone soya broth (TSB) medium at $30^{\circ} \mathrm{C}$. E. coli ET12567 was used for obtaining unmethylated DNA for transformation into S. rimosus. Conjugal transfer of S. rimosus on MS agar was performed as described in earlier study by Kieser et al. (2000). E. coli cultures were grown on LB medium at $37{ }^{\circ} \mathrm{C}$, and chloramphenicol $(25 \mu \mathrm{g} / \mathrm{ml})$, apramycin $(50 \mu \mathrm{g} / \mathrm{ml})$, and kanamycin $(50 \mu \mathrm{g} /$ $\mathrm{ml}$ ) were supplemented into the medium as required.

\section{Isolation and manipulation of DNA}

Molecular manipulations of E. coli and S. rimosus were performed as the methods described in the previous works (Sambrook et al. 1989; Kieser et al. 2000). The plasmids used in this work are shown in Table 1. Total genomic DNA (gDNA) of $S$. rimosus was isolated from a 24-36 h culture in TSB according to the procedure described in the previous work (Hopwood et al. 1985).

\section{Construction of gene mutants in S. rimosus (gene disruption, complementation, and overexpression)}

The disruption mutant was constructed by replacing the response regulator gene $(\operatorname{rim} A 1)$. To construct rimA1 disruption plasmid pKC1139-rim, part of rimA1 gene about 524 bp was amplified with primers DrimF and DrimR (Table 2) from gDNA of S. rimosus M4018. All the primers used in this study are listed in Table 2. After that, the amplified fragment was inserted into the EcoRV site of the pMD19-TS vector, and the fragment was digested by HindIII and XbaI. The resulting DNA fragment was inserted into HindIII and $X b a I$ digested pKC1139 (Bierman et al. 1992) to obtain pKC1139-rim. The recombinant plasmid pKC1139-rim was transformed 
Table 1 Bacterial strains and plasmids used in this study

\begin{tabular}{|c|c|c|}
\hline & Genotype/description & References/source \\
\hline \multicolumn{3}{|l|}{ S. rimosus strains } \\
\hline M4018 & The genetically most characterized strain, the oxytetracycline-producing Pfizer strain & (Rhodes et al. 1984) \\
\hline M4018 (plB-KA) & Integrated a control plasmid pIB-KA in the genome of M4018 & This study \\
\hline M4018 (plB-KA-rim) & rimA1A2-overexpressed strain, derived from M4018 & This study \\
\hline M4018 $\Delta$ rim & rimA1-disrupted mutant, derived from M4018 & This study \\
\hline M4018 $\Delta \operatorname{rim}(p \mid B-K A)$ & Integrated a control plasmid plB-KA in the genome of M4018 $\Delta$ rim & This study \\
\hline M4018 $\Delta$ rim (plB-KA-rim) & RimA1A2-complemented strain, derived from M4018 $\Delta$ rim & This study \\
\hline \multicolumn{3}{|l|}{ E. coli strains } \\
\hline $\mathrm{DH} 5 \mathrm{a}$ & Used for routine subcloning & Novagen \\
\hline ET12567 (pUZ8002) & Donor strain for conjugation between E. coli and Streptomyces & (MacNeil et al. 1992) \\
\hline \multicolumn{3}{|l|}{ Plasmids } \\
\hline pMD19-TS & T vector with ampicillin resistance gene & Takara \\
\hline pET-28a & The donor of the kanamycin resistance fragment & Novagen \\
\hline pKC1139 & $\begin{array}{l}\text { An E. coli-Streptomyces shuttle vector containing the apramycin resistance gene aac(3)IV, } \\
\text { the oriT of plasmid RP4, and a temperature-sensitive replicon }\end{array}$ & (Bierman et al. 1992) \\
\hline pKC1139-rim & For disruption of rimA1 gene, containing part of rimA1 gene & This study \\
\hline plB139 & $\begin{array}{l}\text { attP-int derived integration vector for the conjugal transfer of DNA from E. coli to Streptomy- } \\
\text { ces, containing the apramycin resistance gene } a a c(3) / V \text { and the ermE* promoter }\end{array}$ & (Wilkinson et al. 2002) \\
\hline PIB-KA & Additional kanamycin resistance genes inserted into plB139 & This study \\
\hline pIB-KA-rim & pIB-KA derivative harboring the complete rimA1A2 gene under the control of PermE* & This study \\
\hline
\end{tabular}

into E. coli ET12567 and successively introduced into $S$. rimosus M4018 by intergeneric conjugation (Kieser et al. 2000). pKC1139 contains a temperature-sensitive replicon from S. ghanaensis that only works at temperatures below $34{ }^{\circ} \mathrm{C}$. Apramycin-resistant exconjugants were selected at $37{ }^{\circ} \mathrm{C}$, and the rimA1-disrupted mutant $(S$. rimosus M4018 $\Delta$ rim) was confirmed by PCR with primers rim-single-P1/rim-single-P2, aprF/aprR (Table 2), and product sequencing. The integrative vector $\mathrm{PIB}-\mathrm{KA}$ was generated by inserting kanamycin resistance fragment into the NheI site of the pIB139. This kanamycin resistance fragment contained the entire kanamycin resistance gene and about $100 \mathrm{bp}$ of DNA on both side of the gene including the promotor and transcription terminator region. In addition, this fragment was amplified by PCR using plasmid pET-28a (Novagen, USA) as templates with primers pKANTTF/pKANTTR.

For the construction of complementation plasmid, a 2070 bp DNA fragment containing the entire rimA1/A2 gene was amplified by PCR from the gDNA of M4018 using rim-pIBF and rim-pIBR primers. This fragment was digested by corresponding enzymes and inserted into EcoRI and NdeI digested pIB-KA downstream of the ermE* promoter to obtain pIB-KA-rim. The plasmid was transformed into $S$. rimosus M4018 $\Delta$ rim by conjugation, as mentioned above, apramycin-resistant and kanamycinresistant exconjugants were selected for this. The resulting complementation strain M4018 $\Delta$ rim (pIB-KA-rim) containing an integrated copy of rimA1A2 was confirmed by $\mathrm{PCR}$ with primers rimL/rimR preliminarily. To generate M4018 $\Delta$ rim (pIB-KA) used as control, a demethylated empty plasmid pIB-KA was introduced into M4018 $\Delta$ rim.

The demethylated plasmids pIB-KA-rim and pIB-KA were also transformed into S. rimosus M4018 by conjugation to obtain overexpression strain M4018 (pIBKA-rim) and its corresponding control strain M4018 (pIB-KA). The integration of gene was occurred between the $a t t P$ site located in PIB-KA and the $\Phi C 31$ attachment attB site in the $S$. rimosus chromosome.

New attL fragment (401 bp) and attR fragments (502 bp) were developed after the integration. attL fragment was confirmed by PCR using primers attLF and attPR, and attR fragments were confirmed by PCR using primers attPF and attRR. All the plasmids mentioned were identified by enzyme digestion and DNA sequencing.

Determination of dry cell weight and antibiotic production The spores of S. rimosus were collected, respectively, when all the strains are cultivated on wheat bran medium at $30{ }^{\circ} \mathrm{C}$ for $5-7$ days. The concentrations of spore suspension were determined by dilution method of plate counting. The spore suspension of different strains was inoculated into MM liquid medium supplemented with $50 \mathrm{mM}$ glycine to a final concentration of 
Table 2 Primers used in this study

\begin{tabular}{|c|c|}
\hline Primers & Sequence $\left(5^{\prime}-3^{\prime}\right)$ \\
\hline \multicolumn{2}{|c|}{ PCR amplification } \\
\hline DrimF & CCCAAGCTTACACCTCGACGATGTTGTTG \\
\hline DrimR & TGCTCTAGAATACGCGGTCGATGTGGTG \\
\hline aprF & GTGCAATACGAATGGCGAAAA \\
\hline aprR & TCAGCCAATCGACTGGCGA \\
\hline rim-single-P1 & AACCGGTAGTCCGCCGTACGGTG \\
\hline rim-single-P2 & AGGTTGAGAAGCTGACCGATGAGCTC \\
\hline pKANTTF & CTAGCTAGCCTCAGTGGAACGAAAACTCAC \\
\hline PKANTTR & CTAGCTAGCACAATTTCAGGTGGCACTTTTC \\
\hline rim-plBF & CGGAATTCCTACGCCACCGGCAGGCGTA \\
\hline rim-plBR & GGAATTCCATATGCGCCTGTTGATCGTGGAG \\
\hline riml & CTACGCCACCGGCAGGCGTAGTTC \\
\hline $\operatorname{rimR}$ & ATGCGCCTGTTGATCGTGGAGGAC \\
\hline attLf & GTTCACCCACAGCTGGAGGCC \\
\hline attLr & GCTCGACTTCGCGCTGAAGGT \\
\hline attRf & GCTATAATGACCCCGAAGCAG \\
\hline attRr & TCGTCATGCCCCGCAGT \\
\hline \multicolumn{2}{|l|}{ RT-PCR } \\
\hline co-hrdF & GTCCAGGTAGTCGCGCAGGAC \\
\hline co-hrdR & TCTCGCTGCACACCCCGCTG \\
\hline co-rimF & GTCGCGATCTGCGTGGCGAC \\
\hline co-rimR & TGCTGCGCGTGGGAGAGCTG \\
\hline \multicolumn{2}{|l|}{ RT-qPCR } \\
\hline oxyB-F & ACGTCAGCGCGTATCAGTCCTTC \\
\hline oxyB-R & GCACAGCGAACCGTCCACAC \\
\hline otrB-F & TTCGCCTTCCTCCAGATGGCGTG \\
\hline otrB-R & AGCGGTACGTTGAGCAGGAACAC \\
\hline $\operatorname{otrC}-\mathrm{F} 2$ & TGCTGTATCTGGACGAGCCGAC \\
\hline $\operatorname{otrC}-\mathrm{R} 2$ & GTCGATGACCGTCAGCTCGTG \\
\hline otcG-F & ATGTCATTCTGCTGGACGGGTC \\
\hline otcG-R & TCAGAAATCCGCTCACGCGCAG \\
\hline otcR-F & ATGAAGTCCTTGCGCAGCTGGTAG \\
\hline otcR-R & CTCCGAAAGTGAAGCAGGTGCTC \\
\hline hrdB-F & AGAGCGTCGCAGCGAAGAGTC \\
\hline hrdB-R & СTTCTTGGCGACCGTCTTCTTCTC \\
\hline
\end{tabular}

Restriction sites are underlined

$1.0 \times 10^{6}$ spores $/ \mathrm{ml}$. All fermentation cultures were cultivated at $30{ }^{\circ} \mathrm{C}$ on a rotary shaker $(220 \mathrm{rpm})$ for 7 days. All mutants were undertaken in three parallels to ensure reproducibility. Culture samples were taken after every $24 \mathrm{~h}$.

For dry cell weight determination, $2 \mathrm{ml}$ culture samples at different times were washed twice with ultrapure water and dried at $90{ }^{\circ} \mathrm{C}$ until constant weight is achieved. For an assessment of OTC production, the $\mathrm{pH}$ of fermentation culture samples were adjusted to $1.5-2.0$ with $9 \mathrm{M}$ $\mathrm{HCl}$ and centrifuged at $12,000 \mathrm{rpm}$. After that, a $0.22 \mu \mathrm{m}$ filter (Merck Millipore, USA) was used to filter the supernatant. Subsequently, $20 \mu \mathrm{l}$ sample was analyzed using high-performance liquid chromatography (HPLC, Agilent 1100 HPLC system) equipped with a C18 column $(4.6 \times 200 \mathrm{~nm}$, Kromasil, Sweden). A mix solution containing $10 \%$ phosphoric acid $(0.2 \mathrm{M}), 10 \%$ methanol, $20 \%$ acetonitrile, and $60 \% \mathrm{H}_{2} \mathrm{O}$ was applied with a constant flow rate of $0.8 \mathrm{ml} / \mathrm{min}$ as per the method described by the previous work (Wang and Yang 1995). The corresponding detected peak areas at $350 \mathrm{~nm}$ were used to calculate the concentration of OTC. A standard OTC sample (Sigma, USA) was used to make standard curves.

\section{RNA preparation, RT-PCR and quantitative RT-PCR}

RT-PCR was used to determine whether $\operatorname{rimA1}$ and $\operatorname{rim} A 2$ were co-transcribed, and qRT-PCR was used to determine the transcriptional level of the target genes. Briefly, S. rimosus mycelia were collected and frozen immediately in liquid nitrogen followed by grounding into powdered form. Fresh samples were stored at $-80{ }^{\circ} \mathrm{C}$ after liquid nitrogen freezing for total RNA extraction.

The AxyPrep ${ }^{\mathrm{TM}}$ multisource total RNA miniprep kit (Axygen, USA) was used for total RNA extraction as per the manufacturer's instructions. The concentration and purity of RNA were measured with Nanodrop 2000 (Thermo Scientific, USA) as per the manufacturer's instructions. RNA samples (RNA: protein ratio, $\mathrm{OD}_{260} /$ $\mathrm{OD}_{280}, 1.8-2.0$ ) were screened through gel electrophoresis to check their integrity (Bustin et al. 2009; Taylor et al. 2010). All experiments were performed in triplicates.

For reverse transcription, PrimeScript ${ }^{\mathrm{TM}}$ RT Reagent Kit with gDNA Eraser (TaKaRa, Japan) was used as per the manufacturer's instructions. Nanodrop 2000 was used to analyze the concentration of cDNA. cDNA samples in ten-fold serial dilution was prepared to make a standard curve and each dilution was performed under a range of temperatures around the calculated $\mathrm{Tm}$ of the primers to be used in the experiments (Table 2). All RNA samples without prior reverse transcription performed PCR amplification to exclude the possibility of DNA contamination.

RT-PCR was used to determine whether $\operatorname{rimA1}$ and $\operatorname{rim} A 2$ are co-transcribed by primers co-rimF/co-rimR (Table 2). Gene $h r d B$ in S. rimosus was served as positive internal control for RT-PCR. RNA treated with DNase I was used as a negative control. The reverse transcription procedure was as follows: $94{ }^{\circ} \mathrm{C}$ for $3 \mathrm{~min}, 28$ cycles for $94{ }^{\circ} \mathrm{C} 30 \mathrm{~s}, 62{ }^{\circ} \mathrm{C} 30 \mathrm{~s}, 72{ }^{\circ} \mathrm{C} 30 \mathrm{~s}$. The reaction samples were analyzed by electrophoresis.

SYBR $^{\circledR}$ GC Premix Ex Taq ${ }^{\text {TM }}$ kits (TaKaRa, Japan) was used to perform qRT-PCR according to the manufacturer's instructions. The melting curve analysis was executed 
by a CFX96 ${ }^{\mathrm{TM}}$ real-time PCR detection system (Bio-Rad, USA) (Lu et al. 2007; Rozas et al. 2012; Yu et al. 2012a, b). The optimized Tm and cDNA concentration of each gene were used for qRT-PCR analysis and the Cq values were determined for optimization of qRT-PCR conditions (Taylor et al. 2010).

The relative transcript levels of tested genes were normalized to $h r d B$ and were determined using the 2-DDCT method (Livak and Schmittgen 2001). Error bars indicated the standard deviation from three independent biological replicates. Data were analyzed by $t$ test, ${ }^{*} P<0.05$, significant difference. Three biological samples from the different bacterial cultures were triply amplified in separate $\mathrm{PCR}$ reactions.

\section{Antibiotic susceptibility test}

Susceptibility of the rimA1-disrupted mutant to antimicrobial agents was tested by a disk-diffusion assay on MM with $50 \mathrm{mM}$ glycine. The same amount of spore of M4018 and other mutants was spread on the solid medium, and then, three Oxford cups (6 $\mathrm{mm}$ in diameter) were put on the medium uniformly in one plate. Different kinds of antibiotic with different concentration were added to the Oxford cup, but the volume of the solution added was kept same $(200 \mu \mathrm{l})$. The radii of the inhibition zone were measured after 3 days of incubation. The radius of the inhibition zone from S. rimosus M4018 plate was used as a control.

The antibiotics used for the study included oxytetracycline $(20,50 \mathrm{mg} / \mathrm{ml})$, kanamycin $(2.5,10,25 \mathrm{mg} / \mathrm{ml})$, chloramphenicol $(0.25 .0 .5,1 \mathrm{mg} / \mathrm{ml})$, rifamycin $(2.5$, $10,25 \mathrm{mg} / \mathrm{ml})$, cefotaxime $(2.5,10,25 \mathrm{mg} / \mathrm{ml})$, ampicillin $(2.5,10,25 \mathrm{mg} / \mathrm{ml})$, and erythromycin $(2.5,10,25 \mathrm{mg} /$ $\mathrm{ml})$.

\section{OTC production under different environmental stresses} To evaluate the OTC production of all the $S$. rimosus mutants grown under various environmental stresses, the liquid $\mathrm{MM}$ with $50 \mathrm{mM}$ glycine containing each of the following chemicals $\mathrm{KCl}(0.1 \mathrm{M})$ and $\mathrm{H}_{2} \mathrm{O}_{2}(2 \mathrm{mM})$ was prepared. The spore suspensions of different mutants were inoculated into these media to a final concentration of $1.0 \times 10^{6}$ spores $/ \mathrm{ml}$, respectively. All fermentation cultures were cultivated at $30{ }^{\circ} \mathrm{C}, 220 \mathrm{rpm}$ for 7 days. Samples were taken from the $250 \mathrm{ml}$ shake flasks once a day and the concentration of OTC was determined by HPLC.

\section{Results}

Two-component systems in S. rimosus and identification of $\operatorname{RimA1A2}$

Total size of $S$. rimosus M4018 genome is estimated to be $9.2 \mathrm{Mb}$ with a $\mathrm{G}+\mathrm{C}$ content of $71.96 \%$. The draft genome of $S$. rimosus M4018 is estimated to have a total of 8584 protein-coding genes, which is largely in agreement with genome of S. rimosus ATCC 10970, the wild-type strain, and in agreement with Pethick et al. (2013). Putative open reading frames (ORFs) and the corresponding proteins in the M4018 strain genome were annotated based on the PFAM database (Finn et al. 2016). 77 putative response regulators, containing the response-reg domain and 177 putative histidine kinase homologs including Hpt, HisKA, HisKA_3, HATPase_c_2, and HATPase_c domain, were identified in the genome of M4018 strain (data not shown). Phylogenetic analysis was established by applying software MEGA 7 with Neighbor-Joining method to analyze the 77 response regulators with response reg domain (Kumar et al. 2016). According to our analysis, the most RRs identified in the genome of M4018 strain belong to the OmpR and NarL families (Fabret et al. 1999; Hutchings et al. 2004) (Fig. 1). 50 pairs of typical TCS comprising a histidine kinase and a cognate response regulator protein were screened, considering that HK and RR of a typical TCS are adjacent to each other on the chromosome, and they are generally transcribed in the same orientation.

Comparative analysis of 50 typical TCSs identified in the M4018 genome with all the TCSs of S. coelicolor published in the literature also identified the orf04661 (RR) and orf04662 (HK) TCS homologues, with $82 \%$ and $71 \%$ identity to RapA1 (RR) and RapA2 (HK) in S. coelicolor at amino acid sequence level, respectively ( $\mathrm{Lu}$ et al. 2007). It was demonstrated that TCS homologue RapA1A2 in $S$. coelicolor is involved in the regulation of secondary metabolite biosynthesis ( $\mathrm{Lu}$ et al. 2007). Based on this information, we aimed to evaluate the potential role of RapA1A2-like homologues orf04661 (RR) and orf04662 (HK), designated here as RimA1A2 in OTC biosynthesis and in potential physiological and morphological properties of the 4018 strain under different culture conditions.

\section{Sequence analysis of the $\operatorname{RimA} 1$ and $\operatorname{RimA} 2$ in S. rimosus M4018}

Genes $\operatorname{rim} A 1$ and $\operatorname{rim} A 2$ are adjacent to each other on the M4018 chromosome, and they are transcribed in the same orientation (Fig. 2). The $\operatorname{rim} A 1$ gene encodes a 220 aa protein with a deduced molecular mass of $23.8 \mathrm{kDa}$. It is identified as the response regulator of OmpR family at amino acid level (Hutchings et al. 2004). It contains several conserved amino acids on the $\mathrm{N}$ terminal region, which are required for the formation of phosphorylation pocket including Asp-8, Asp-51 (the putative phosphorylation site), and Lys-101 (Stock et al. 1989). It is suggested that the DNA-binding pocket containing DNAbinding motif, located on the C-terminus of the protein, mediates the direct involvement in the regulatory activity, by binding on the target DNA sequence (Bijlsma and 


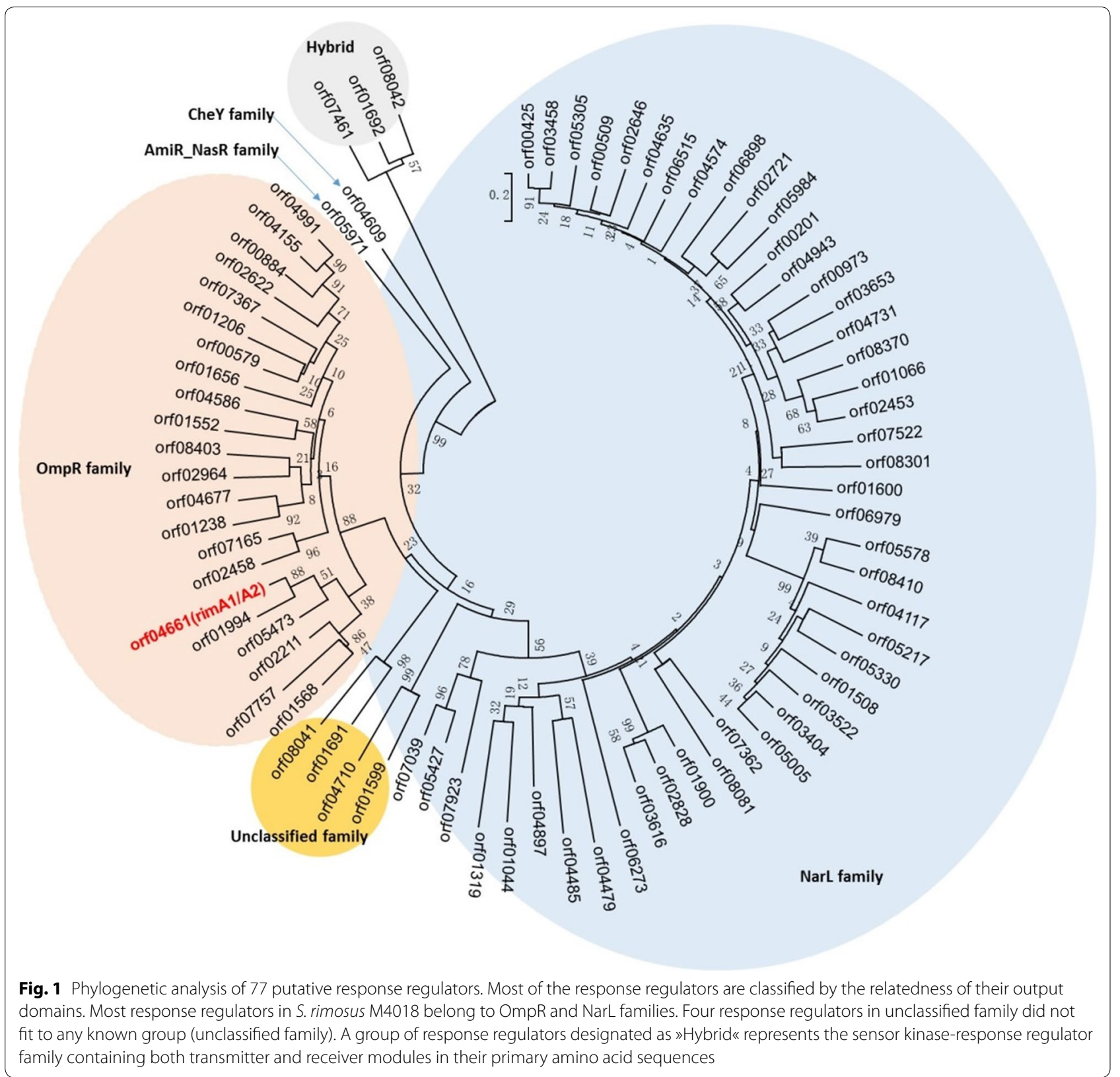

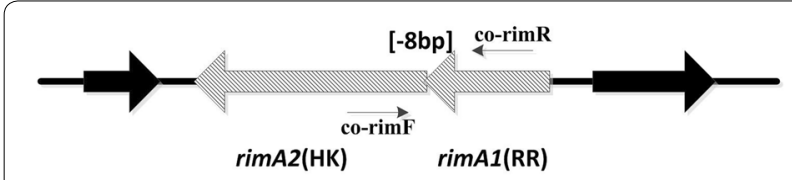

Fig. 2 Organization of the rimA1/A2 genes in the S. rimosus M4018 genome. The orientation of the rimA1/A2 transcription is indicated by arrows. Numbers in square brackets represent the length of intergenic regions in bp

Groisman 2003). The gene product encoded by rimA2 is a putative 467 aa protein with a deduced molecular mass of $49.7 \mathrm{kDa}$. Hypothetical transmembrane domain is identified in its amino-terminal region, which may serve as an anchor to the cytoplasmic membrane. The deduced RimA2 protein displays typical properties of the sensory protein of prokaryotic histidine kinases, containing the $\mathrm{H}$ box (His-271 being the putative phosphorylation site) and motifs N, G (Hoch and Silhavy 1995; Kim and Forst 2001; Bijlsma and Groisman 2003).

The stop and start codons of $\operatorname{rim} A 1$ and $\operatorname{rim} A 2$, respectively, overlap by 8 bp (Fig. 2), strongly suggesting that both genes are co-transcribed. We have performed RTPCR assay with a pair of primers (co-rimF and co-rimR, Table 2) to determine whether the two genes are form an 
operon or not. Primer co-rimF is located at the last 155$180 \mathrm{bp}$ from the $3^{\prime}$-end of rimA 2 and primer co-rimR is located at the first 265-290 bp of the $5^{\prime}$-end of the downstream gene rimA1 (Fig. 2). Expected size (458 bp) of the PCR product, corresponding to the expected DNA fragment containing part of $\operatorname{rim} A 1 / A 2$, was detected (Additional file 1: Figure S1), suggesting that the two genes were co-transcribed as a single-polycistronic mRNA.

\section{RimA1A2 system regulates antibiotic production in a medium-dependent manner in S. rimosus M4018}

The TCS RapA1/A2, which regulates the biosynthesis of both actinorhodin and a type I polyketide in S. coelicolor, was first identified by Lu et al. using genetic and proteomic approaches (Lu et al. 2007). Although no growth and morphological difference was shown between the rapA1/A2 knockout mutant and wild-type strain S. coelicolor M145, a distinct decrease in the production of ACT was observed in rapA1/A2 mutant when cultivated in rich $\mathrm{R} 4 \mathrm{C}$ agar or $\mathrm{R} 4 \mathrm{C}$ liquid media.

In this study, we constructed a rimA1-disrupted mutant of S. rimosus 4018 (M4018 $\Delta \mathrm{rim}$ ). Compared with the parental strain S. rimosus M4018, the disruption mutant M4018 $\Delta$ rim showed no visible change in the culture pigmentation, OTC biosynthesis, morphology, or any other phenotype when cultivated on rich media such as TSB (Fig. 3), R4C, MS, and R2YE (Hopwood et al. 1985) (data not shown).

OTC biosynthesis, which is catalyzed by the type II polyketide synthase (PKS), has been extensively studied (Petkovic et al. 2017). OTC production is well correlated with pigment formation in the culture of $S$. rimosus strains, considering that OTC itself is pigmented compound (Petkovic et al. 2006). Therefore, relative yield of OTC on a solid agar plate or in a liquid medium correlates roughly to the intensity of the orang-brown pigment. Pigmentation and OTC production, however, also depends upon the composition of medium.

Often, the phenotypic difference between the parental strain and its mutant could only be observed at specific cultivation conditions, such as cultivation on minimal medium (MM) or medium with different sources of carbon, nitrogen, or phosphate (Sola-Landa et al. 2003; Shu et al. 2009; Yu et al. 2012a, b). Therefore, we set up testing procedure, where we carried out comparative evaluation of the morphological properties of rimA1-disrupted mutant and parent strain M4018 in an MM medium supplemented with various medium components on the agar medium plate assay. Initially, the glucose was replaced by different carbon sources such as D-mannitol, lactose, arabinose, starch (gelatinized before use), and maltose. Independently on the carbon source, the molar concentration of carbon was maintained at $330 \mathrm{mM}$, considering

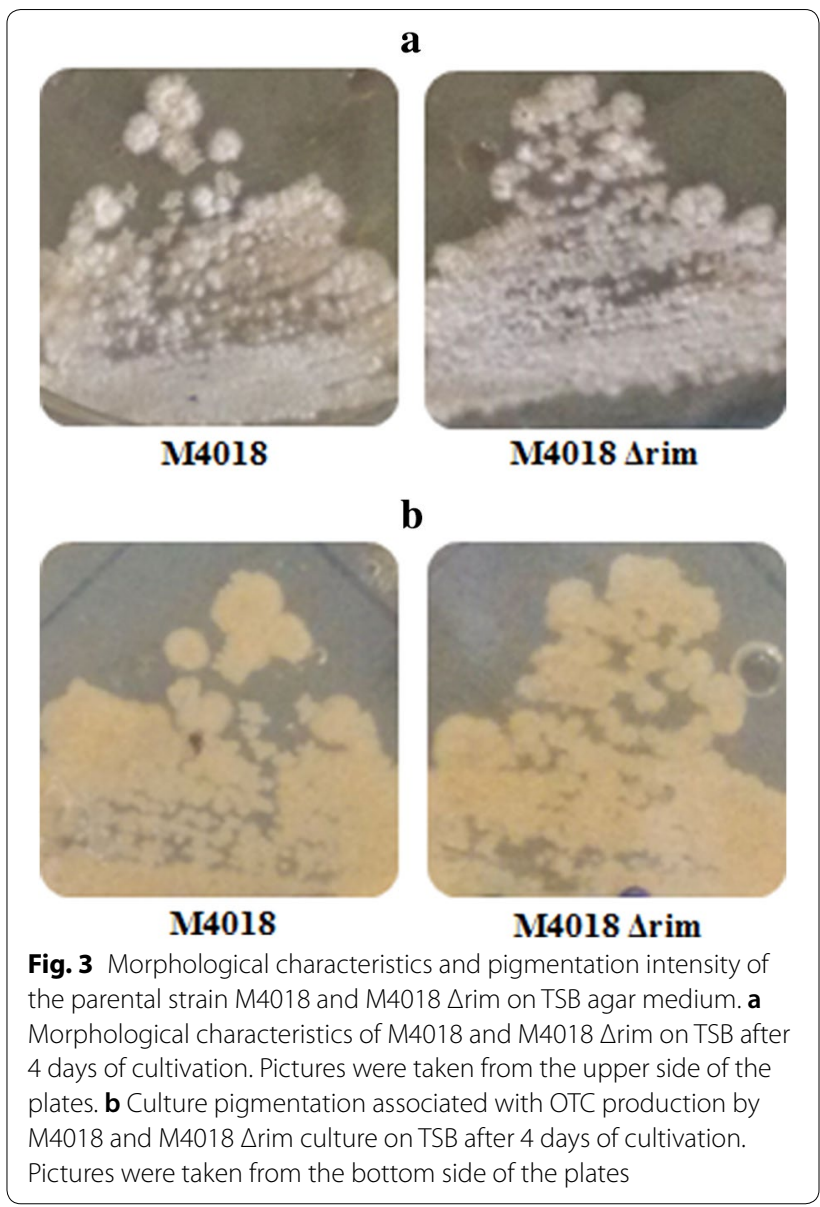

that MM containing $10 \%$ glucose $(55 \mathrm{mM})$ was used as control. After 4 day cultivation, no significant morphological difference between S. rimosus M4018 and the rimA1-disrupted mutant was observed on any medium tested (Additional file 1: Figure S2). In the second step, we carried out testing procedure in a plate assay on an MM medium supplemented with different organic nitrogen sources (Shu et al. 2009; Yu et al. 2012a, b). Nine nitrogen sources, including amino acids glycine (Gly), threonine (Thr), proline (Pro), asparagine (Asn), aspartate (Asp), glutamate (Glu), glutamine (Gln), lysine (Lys), and alanine (Ala), were tested, and their concentrations in the MM medium were adjusted to $50 \mathrm{mM}$. Interestingly, we observed significant increase in pigmentation intensity with rimA1-disrupted strain when cultivated on $\mathrm{MM}$ with $50 \mathrm{mM}$ Gly as the sole nitrogen source, and no difference was observed when cultivated on MM supplemented with $50 \mathrm{mM}$ Asn (Additional file 1: Figure S3), thus indicating that RimA1A2 may negatively regulate OTC biosynthesis. Actually, these nine nitrogen sources were divided into three groups. In the first group, including Lys, Ala, Asn, and Glu, no visible difference was observed when the nitrogen source is added into MM. 
In the second group, including Thr, Pro, and Gln, the visible difference was observed when the nitrogen source is added into MM, but the relative OTC production was low. In the third group, including Asp, Gly, the visible difference was observed when the nitrogen source is added into MM, and the relative OTC production was high. The most significant difference in pigment production was observed when MM was supplemented with Gly among all the nitrogen sources.

In the next step, we aimed to evaluate potential role of RimA1A2 in the regulation of OTC production in the liquid culture. S. rimosus M4018 and the rimA1-disrupted mutant (M4018 $\Delta$ rim) were cultivated in the liquid MM supplemented with $50 \mathrm{mM}$ glycine. MM supplemented with $50 \mathrm{mM}$ Asn was used as control. M4018 $\Delta$ rim and M4018 showed the identical growth rate and OTC titer when cultivated in liquid MM supplemented with $50 \mathrm{mM}$ Asn (Fig. 4a). The rimA1-disrupted strain showed a slightly increased growth rate when cultivated in liquid MM supplemented with $50 \mathrm{mM}$ Gly. However, disruption of rimA1 resulted in significant increase of OTC production in liquid MM supplemented with $50 \mathrm{mM}$ Gly. OTC titer of the rimA1-disrupted strain was increased by $63 \%$, compared to that of M4018 after 5 days of cultivation (Fig. 4b).

To make sure that the phenotypic changes observed in the rimA1-disrupted strain were not caused by a polar effect, so we carried out in-trans complementation of the entire rimA1A2 expressed under strong ermE* promoter in the rimA1-disrupted strain using the ФC31based integrative vector PIB-KA. The complementation of the rimA1-disrupted strain with the entire $\operatorname{rimA1A2}$ expressed under strong ermE ${ }^{*}$ promoter reduced OTC production of the disruption mutant cultivated on MM supplemented with $50 \mathrm{mM}$ glycine. Nevertheless, the complementation mutant M4018 $\Delta$ rim (pIB-KA-rim)
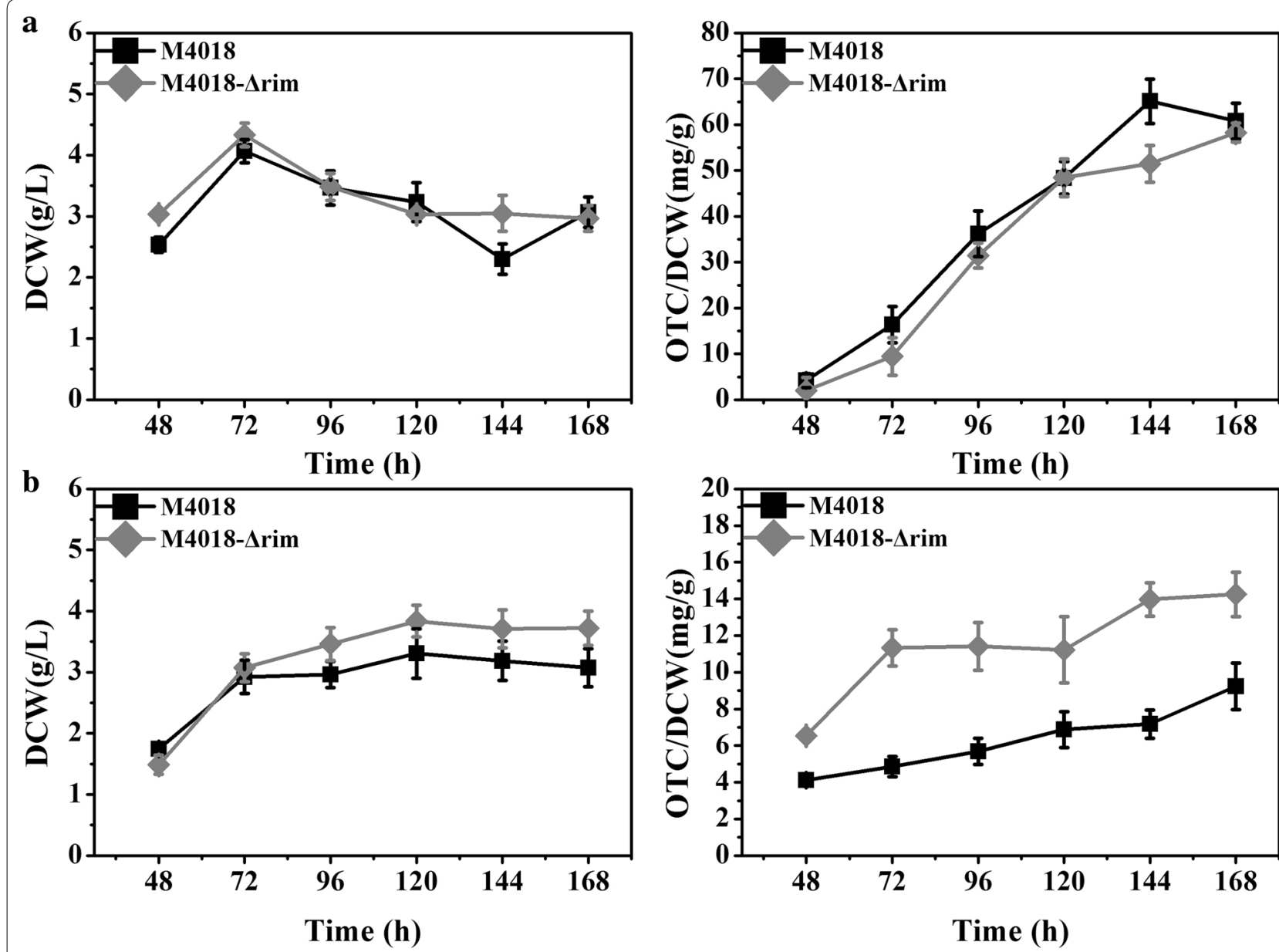

Fig. 4 Biomass formation and OTC production of M4018 (black square) and M4018 $\triangle$ rim (black diamond) cultivated on MM medium supplemented with 50 mM Asn (a) or Gly (b), respectively. The data represent mean values and standard deviation of three independent experiments 
still produced more OTC compared to the parent M4018 strain containing an extra copy of rimA1A2 expressed under strong ermE $^{*}$ promoter [M4018 (pIB-KA-rim)] at the end of the culture ( $t>144 \mathrm{~h}$ ) (Fig. 5), which is an additional confirmation that RimA1A2 act as a negative regulator when cultivated on MM supplemented with Gly.

We have not observed any significant difference in OTC production when comparing M4018 (pIB-KA) and M4018, or comparing M4018 $\Delta$ rim and M4018 $\Delta$ rim (pIB-KA) strains, indicating that the introduction of the empty pIB-KA plasmid had no effect on the OTC production in M4018 parent and M4018 $\Delta$ rim strains. We can, therefore, conclude that the increase in OTC production was really due to the disruption of the $\operatorname{rimA} 1$ gene which further confirms that the RimA1A2 negatively regulates OTC biosynthesis.

\section{Role of RimA1A2 in the physiological properties of $S$. rimosus 4018}

As mentioned earlier, two-component regulatory elements are very abundant in secondary metabolite-producing organisms such as $S$. rimosus. However, limited data are available in the literature on the wider environmental aspect of TCSs, and their role in diverse physiological processes and morphological differentiation in filamentous organisms such as Streptomyces species. Among the presumptive signals, the potentially sensed

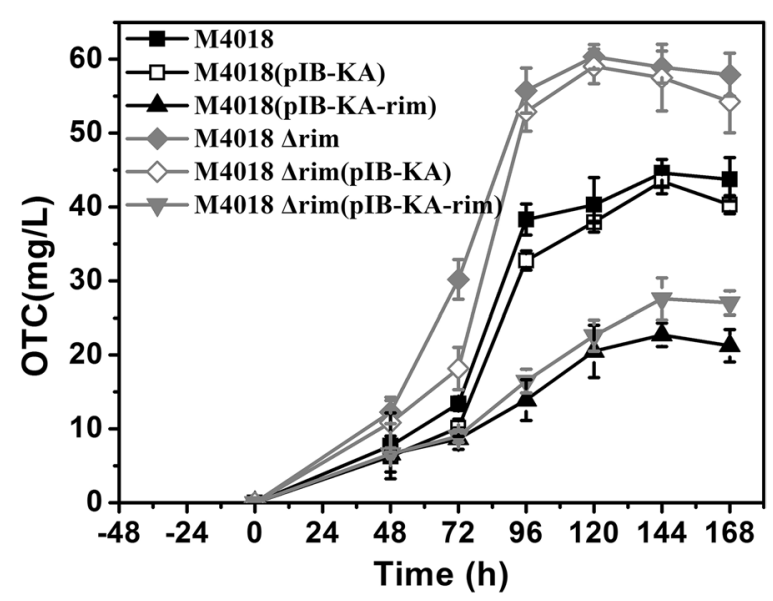

Fig. 5 OTC production by M4018 and the engineered strains cultivated on MM medium supplemented with 50 mM Gly. Vertical error bars correspond to the standard error of the mean of three replicated cultures. Black square: parental strain M4018; white square: parental strain M4018 with a control plasmid pIB-KA; black up-pointing triangle: rimA1A2-overexpressed mutant M4018 (plB-KA-rim); black diamond: rimA1-disrupted mutant M4018 Arim; white diamond: M4018 $\triangle$ rim with a control plasmid pIB-KA; black down-pointing triangle: rimA1A2-complemented mutant M4018 $\Delta$ rim (plB-KA-rim) by TCS are the chemical and physical parameters such as presence or lack of different ions, temperature and $\mathrm{pH}$ value variation, the redox state of electron carriers in the environment, oxygen pressure, osmolality, and many other culture parameters (Bang et al. 2000; Ahn et al. 2012; Djoric and Kristich 2015). Therefore, we also attempted to determine the role of response regulator RimA1 to the exposure of S. rimosus against different antibiotics in the environment. Conclusively, we evaluated the potential role of RimA1 in the regulation of OTC production under different environmental stresses conditions including increased osmolality and oxidative stress. The applied approach in this study provides us more comprehensive understanding of the role of TCS such as RimA1A2 on environmental adaptation and antibiotic production.

\section{Evaluating the role of response regulators in antibiotic resistance}

The disk-diffusion assay was applied to determine the degree of sensitivity of the rimA1-disrupted mutant to the selected antibiotics compared to the M4018 parent strain. The spores of M4018 and M4018 $\Delta$ rim were spread on the solid medium plate and the antibiotic solutions were added into the Oxford cup. The diameter of inhibition zone was inversely proportional to the resistance, as described in "Materials and methods". We have observed that the self-resistance of M4018 $\Delta$ rim against OTC was slightly enhanced (about 10-15\%) (Table 3). This phenomenon corresponded to the increase of OTC production of the rimA1-disrupted mutant. In comparison with the parental strain, M4018 $\Delta$ rim also showed increased resistance to kanamycin (about 30\%) and chloramphenicol (about 25\%) (Table 3). The resistance of the M4018 $\Delta$ rim strain to the remaining panel of antibiotics was not significantly different compared to M4018 strain.

\section{Biosynthesis of OTC by the rimA1-disrupted mutant under different environmental stresses conditions}

The response regulator mutants were tested for its ability to produce OTC under different environmental stresses conditions including increased osmolality (addition of $\mathrm{KCl}$ ) and oxidative stress (addition of $\mathrm{H}_{2} \mathrm{O}_{2}$ ). The disruption mutant M4018 $\Delta$ rim showed increased OTC production level in liquid MM supplemented with $50 \mathrm{mM}$ glycine. Under identical cultivation conditions, the overexpression of $\operatorname{rim} A 1 A 2$ in the strain containing the second copy of $\operatorname{rim} A 1 A 2$ expressed under strong constitutive $e r m E^{*}$ promoter resulted in significant decrease in OTC production (Fig. 6a). In general, $\mathrm{KCl}$ has been extensively used as an osmotic stress-inducing agent in E. coli, Streptomyces and other bacteria (Sugiura et al. 1994; Wood 1999; 
Table 3 Inhibition zone radius of M4018 and M4018 $\Delta$ rim

\begin{tabular}{|c|c|c|}
\hline \multirow[t]{2}{*}{ Antibiotics } & \multicolumn{2}{|c|}{ Inhibitory zone radius/mm } \\
\hline & M4018 & M4018 $\Delta$ rim \\
\hline \multicolumn{3}{|l|}{ OTC (mg/ml) } \\
\hline 20 & $10.33 \pm 0.58$ & $9.33 \pm 0.58$ \\
\hline 50 & $15.67 \pm 1.15$ & $13.67 \pm 1.15$ \\
\hline \multicolumn{3}{|l|}{$\operatorname{Kan}(\mathrm{mg} / \mathrm{ml})$} \\
\hline 2.5 & $11.33 \pm 0.58$ & $8.67 \pm 0.58$ \\
\hline 10 & $16.67 \pm 1.15$ & $12.33 \pm 1.15$ \\
\hline 25 & $19.67 \pm 1.53$ & $15.00 \pm 1.00$ \\
\hline \multicolumn{3}{|l|}{ Cef $(\mathrm{mg} / \mathrm{ml})$} \\
\hline 2.5 & - & - \\
\hline 10 & $6.00 \pm 1.00$ & $6.33 \pm 0.58$ \\
\hline 25 & $12.00 \pm 0.00$ & $11.67 \pm 1.53$ \\
\hline \multicolumn{3}{|l|}{ Ery $(\mathrm{mg} / \mathrm{ml})$} \\
\hline 2.5 & $15.67 \pm 1.15$ & $16.00 \pm 1.73$ \\
\hline 10 & $18.00 \pm 1.00$ & $17.33 \pm 1.15$ \\
\hline 25 & $21.00 \pm 1.00$ & $20.33 \pm 1.25$ \\
\hline \multicolumn{3}{|l|}{ Amp (mg/ml) } \\
\hline 2.5 & - & - \\
\hline 10 & $8.67 \pm 1.53$ & $8.67 \pm 0.58$ \\
\hline 25 & $12.67 \pm 0.58$ & $12.33 \pm 1.15$ \\
\hline \multicolumn{3}{|l|}{ Chl (mg/ml) } \\
\hline 0.25 & $17.67 \pm 0.58$ & $14.33 \pm 1.15$ \\
\hline 0.5 & $21.00 \pm 1.00$ & $17.00 \pm 0.00$ \\
\hline 1 & $25.33 \pm 0.58$ & $20.00 \pm 1.73$ \\
\hline \multicolumn{3}{|l|}{$\operatorname{Rifa}(\mathrm{mg} / \mathrm{ml})$} \\
\hline 2.5 & $17.67 \pm 0.58$ & $17.33 \pm 1.53$ \\
\hline 10 & $20.67 \pm 1.15$ & $19.00 \pm 1.00$ \\
\hline 25 & $21.67 \pm 1.53$ & $21.33 \pm 1.53$ \\
\hline
\end{tabular}

Numbers in italic represent significant increase in radius of inhibitory zone under the same experimental conditions. M4018: the parental strain, M4018 $\Delta$ rim: the rimA1-disrupted mutant derived from M4018; the antibiotics used in the study include oxytetracycline (OTC), kanamycin (Kan), cefotaxime (Cef), erythromycin (Ery), ampicillin (Amp), chloramphenicol (Chl) and rifamycin (Rifa)

Bishop et al. 2003). Under osmotic stress caused by the addition of $\mathrm{KCl}$, however, the production of OTC with all tested strains was reduced. The reduction in OTC yield varied depending upon the strain tested (Fig. 6b). The yield of OTC was evaluated at the 6th day of cultivation, considering that OTC yield generally reached the highest value after 6 days of cultivation (Fig. 6). As described in "Materials and methods", after the addition of $\mathrm{KCl}$ into $\mathrm{MM}+\mathrm{Gly}$ at concentration of $0.1 \mathrm{mM}$, we observed the reduction of the final yield of OTC by $78 \%$ with M4018 $\Delta$ rim (pIB-KA) strain. On the other hand, when the control strain M4018 (pIB-KA) containing empty vector was tested, we observed $39 \%$ reduction in the OTC yield. Finally, only $21 \%$ reduction in the final OTC yield was observed in the culture of the parent strain containing the second copy of rimA1A2 expressed under strong promoter ermE* (Fig. 6b, Table 4). Although overexpression of RimA1A2 negatively regulated OTC production in liquid medium $\mathrm{MM}+$ gly with 4018 parent strain, the overexpression of rimA1A2 resulted in increase in yield of OTC when cultivated under adverse osmotic pressure condition. We, therefore, conclude that in relation with OTC biosynthesis, the overexpression of RimA1A2 plays positive role in osmotic stress adaptation.

Besides, the rimA1-disrupted mutant was also tested for its ability to produce OTC under oxidative stress, which is induced by $\mathrm{H}_{2} \mathrm{O}_{2}$ (Beites et al. 2011; Ahn et al. 2012). $\mathrm{H}_{2} \mathrm{O}_{2}$ was added to the liquid $\mathrm{MM}+$ gly before inoculation at the final concentration of $2 \mathrm{mM}$. When M4018 (pIB-KA), M4018 (pIB-KA-rim), M4018 $\Delta$ rim (pIB-KA), and M4018 $\Delta$ rim (pIB-KA-rim) were cultivated in $\mathrm{MM}+$ gly supplemented with $\mathrm{H}_{2} \mathrm{O}_{2}$, the oxidative stress resulted in the partial loss of OTC production to all the strains. However, it caused reduction in the final yield of OTC, depending on the strain tested (Fig. 6c, Table 4). The final yield of OTC of the M4018 $\Delta$ rim (pIB-KA) was reduced by $33 \%$ under oxidative stress, compared to the control strain M4018 (pIB-KA) and rimA1-overexpressed strain M4018 (pIB-KA-rim), reaching reduction of OTC titer of $54 \%$ and $76 \%$, respectively (Table 4). Based on these results, we can conclude that the rimA1-disrupted mutant M4018 $\Delta$ rim (pIB-KA) exhibited the highest oxidative stress tolerance in relation with the OTC production, in contrast to the osmotic stress caused by $\mathrm{KCl}$ addition, where the rimA1-disrupted strain showed the highest osmotic stress related to the OTC production. We have thus demonstrated that the RimA1A2 has a role in the regulation of OTC production in response to different environmental stresses.

\section{Role of RimA1A2 in regulation of OTC biosynthesis}

Compared with M4018 parent strain, OTC titer of the rimA1-disrupted mutant was significantly enhanced in MM supplemented with $50 \mathrm{mM}$ glycine. The main cause for increase in OTC production by M4018 $\Delta$ rim (pIBKA) strain could be consequence of changes in metabolic supply of building blocks, used in OTC biosynthesis, malonyl-CoA in this case, and/or in overexpression of genes involved in OTC biosynthesis. OTC is a polyketide secondary metabolite, like many of secondary metabolites produced by Streptomyces; the genes carrying out regulation of OTC biosynthesis are most often grouped to form a uniform gene cluster (Pickens and Tang 2010). Genes $\operatorname{oxy} B$, otrB, otcG, and otcR from the OTC gene cluster and OTC resistance gene otrC located outside the cluster (Yu et al. 2012a, b) were selected for further investigation of transcriptional profile of target genes by qRT-PCR and to 

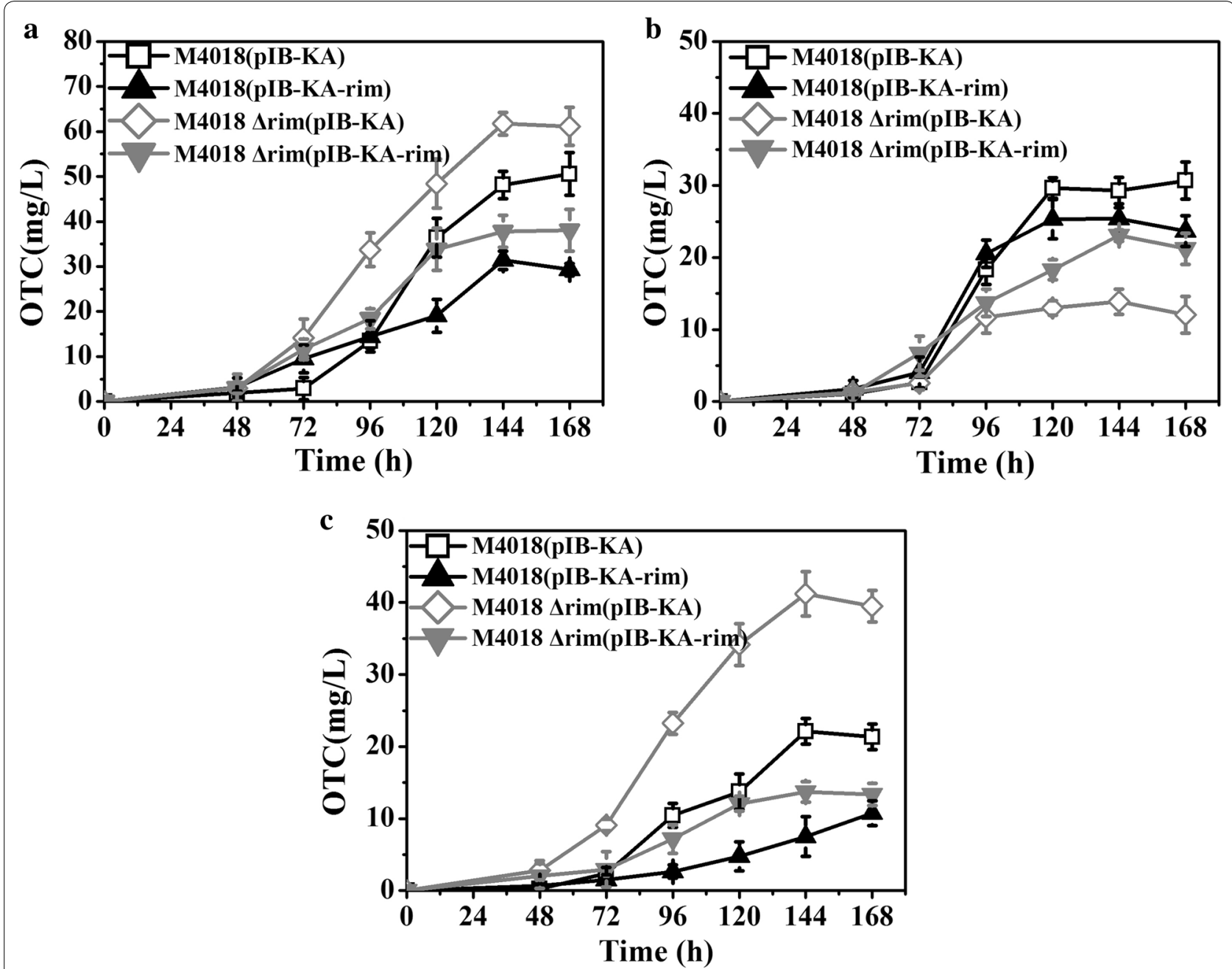

Fig. 6 OTC production by M4018 (pIB-KA) (white square), M4018 (plB-KA-rim) (black up-pointing triangle), M4018 $\Delta$ rim (plB-KA) (white diamond) and M4018 $\triangle$ rim (plB-KA-rim) (black down-pointing triangle) cultivated on MM with 50 mM Gly (a), MM with 50 mM Gly and 0.1 M KCl (b) or MM with $50 \mathrm{mM}$ Gly and $2 \mathrm{mM} \mathrm{H}_{2} \mathrm{O}_{2}$ addition (c). Vertical error bars correspond to the standard error of the mean of three replicated cultures

Table 4 Reduction of OTC production under osmotic or oxidative stress condition

\begin{tabular}{lllll}
\hline & $\begin{array}{l}\text { M4018 } \\
\text { (plB-KA) }\end{array}$ & $\begin{array}{l}\text { M4018 (pIB- } \\
\text { KA-rim) }\end{array}$ & $\begin{array}{l}\text { M4018 } \Delta \text { rim } \\
\text { (plB-KA) }\end{array}$ & $\begin{array}{l}\text { M4018 } \Delta \text { rim } \\
\text { (plB-KA-rim) }\end{array}$ \\
\hline $\mathrm{KCl}(\%)$ & 39 & 21 & 78 & 38 \\
$\mathrm{H}_{2} \mathrm{O}_{2}(\%)$ & 54 & 76 & 33 & 64 \\
\hline
\end{tabular}

M4018 (plB-KA): parental strain M4018 with a control plasmid pIB-KA; M4018 (pIB-KA-rim): rimA1A2-overexpressed mutant derived from M4018; M4018 $\Delta$ rim (plB-KA): rimA1-disrupted mutant M4018 $\Delta$ rim with a control plasmid plB-KA; M4018 $\Delta$ rim (plB-KA-rim): rimA1A2-complemented mutant derived from M4018 $\Delta$ rim

determine the effect of RimA1A2 on the expression profile of genes located in the $o x y$ gene cluster.

While $o x y B$ is a member of so-called minimal PKS, catalyzing formation of nascent polyketide chain (Pickens and Tang 2010), otrB encodes a membrane protein engaged in transport the intracellular OTC out of the cell thus ensuring sufficient self-resistance (Ohnuki et al. 1985; McMurry and Levy 1998). otcG plays a role of conditionally positive regulatory gene in OTC biosynthesis (Lesnik et al. 2009). On the other hand, otcR encodes a pathway-specific regulator that directly activates five promoters from oxy cluster (Yin et al. 2015). The only gene located outside otc gene cluster is the resistance gene $o t r C$, identified by $\mathrm{Yu}$ et al. as an $\mathrm{ABC}$ transporter with multidrug resistance function ( $\mathrm{Yu}$ et al. 2012a, b). As observed in the literature, all the selected five genes have a positive role in OTC production when over-expressed.

The control strain M4018 (pIB-KA), the rimA1disrupted mutant M4018- $\Delta$ rim (pIB-KA), the overexpression mutant M4018 (pIB-KA-rim), and the complemented mutant M4018 $\Delta$ rim (pIB-KA-rim) were 
used to carry out comparative transcriptional analysis of these five selected genes. As shown in Fig. 7, when $\operatorname{rim} A 1$ gene was disrupted, the transcriptional level of all five genes increased significantly. However, observed increase of gene expression varied, when evaluated after $96 \mathrm{~h}$ of cultivation in MM supplemented with Gly. On the other hand, we have observed reduction of the transcriptional level of all five genes when the $\operatorname{rim} A 1 A 2$ was overexpressed in trans under strong constitutive ermE* promoter.

In the control experiment, the entire operon RimA1A2 was used to complement the disrupted mutant strain M4018 $\Delta$ rim, and the transcriptional levels of the genes under investigation in these strains were similar to the M4018 parent strain with chromosomally integrated empty plasmid pIB-KA (Fig. 7). Therefore, we have demonstrated that disruption of $\operatorname{rim} A 1$ led to overexpression of biosynthetic genes of the oxy gene cluster.

\section{Discussion}

RimA1 A2, a distinctive two-component system in S.

\section{rimosus $\mathrm{M} 4018$}

The genome sequence of Streptomyces has revealed a large proportion of regulatory genes, for example, approximately $12 \%$ of the total ORFs in S. coelicolor (Bentley et al. 2002). The first complete genome

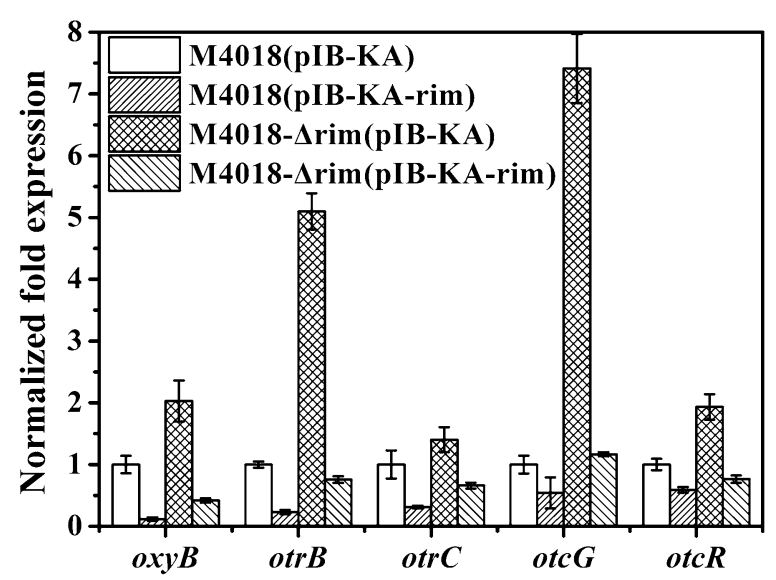

Fig. 7 Evaluation of the transcript levels of five genes oxyB, otrB, otcG, otcR and otrC by qRT-PCR of comparative analysis of the control strain M4018 (plB-KA) (white square), the mutant strains M4018 (pIB-KA-rim) (square with upper right to lower left fill), M4018 $\triangle$ rim (plB-KA) (square with diagonal crosshatch fill) and M4018 $\triangle$ rim (pIB-KA-rim) (square with upper right to lower left fill) when cultivated on MM with $50 \mathrm{mM}$ gly $(96 \mathrm{~h})$. Genes expression in each sample were calculated as the fold expression ratio after normalization to the $\mathrm{hrdB}$ transcript as an internal control. The values are averages of three independent QRT-PCR experiments, error bars indicate standard deviation of model organism S. coelicolor allowed us to analyze typical TCSs, unpaired HKs and orphan RRs (Bentley et al. 2002) and 67 typical TCSs have been found in this organism.

Nearly, all the studied TCSs, which have been characterized in Streptomyces, derived from S. coelicolor (Rodriguez et al. 2013). Although these regulatory elements are widespread throughout the bacteria, little is known about the signals sensed by HK or the downstream binding site of RR in each system, and relatively little is known about their function in secondary metabolism. However, so many TCSs in the S. coelicolor genome clearly imply their important physiological roles. To best of our knowledge, not a single TCS has been characterized in S. rimosus. Most likely, this might happens due to lack of genomics data, and no connection between OTC biosynthesis and potential function of TCSs has been observed to date. Analysis of the S. rimosus M4018 genome revealed about 50 pairs of typical TCSs (Fig. 1). We have identified TCS homologue, designated as RimA1A2, which shows high identity to RapA1A2 from S. coelicolor. In $S$. coelicolor, RapA1A2 regulates the biosynthesis of ACT and an uncharacterized type I polyketide, and the regulatory function exerted by RapA1A2 was dependent on known pathway-specific regulatory genes for these two biosynthetic gene clusters (Lu et al. 2007). Considering that RapA1A2 from $S$. coelicolor exerts positive regulatory role in actinorhodin biosynthesis, we have selected one of the closest homologues designated as RimA1A2, and in continuation, we carried out comprehensive effort to evaluate potential role of this TCS in OTC biosynthesis. The analysis of amino acid sequence confirmed that RimA1 from S. rimosus M4018 is an OmpR family response regulator and RimA2 was a typical histidine kinase.

Considering that OTC is medically important antibiotic, and it is produced at large scale by the industrial fermentation processes, most of the studies in the past have been focused predominantly on cultivation conditions which are comparable or even identical to the cultivation conditions used in industrial environment (Singh et al. 2012; Elsayed et al. 2015). In our study, however, we took different approach. We not only focus on OTC biosynthesis, but also describe the potential role of RimA1A2 on different physiological and morphological properties of S. rimosus.

RimA1A2 exerted negative regulation of OTC biosynthesis in a medium-dependent manner

Similarly, as reported in S. coelicolor, when TCS AfsQ1Q2 (Shu et al. 2009) and DraRK (Yu et al. 2012a, b) were studied, we could not observe any obvious difference in OTC production, or any other phenotypic 
property of $S$. rimosus culture when evaluating rimA1disrupted mutant in media regularly used for cultivation of S. rimosus, such as rich media TSB (Fig. 3), R4C or MS (Hopwood et al. 1985). Yu et al. demonstrated changes in culture pigmentation when $S$. coelicolor disrupted mutant $\triangle d r a R K$ was cultivated on MM containing increased concentration of nitrogen sources such as Glu, Gln, Thr, and Gly or mineral sources of nitrogen such as $\mathrm{KNO}_{3}$ and $\left(\mathrm{NH}_{4}\right)_{2} \mathrm{SO}_{4}$. Importantly, some studies have also observed the significant reduction in actinohrodin production. In contrast, biosynthesis of undecylprodigiosin (RED) was drastically increased (Yu et al. 2012a, b). We, therefore, assumed that RimA1A2 regulation of OTC biosynthesis in $S$. rimosus may be observed in a medium-dependent manner. Therefore, we make our efforts on testing different kinds of media. Hence, we tested different carbon and nitrogen sources in MM, the most obvious phenotypic changes to rimA1-disrupted mutant were observed in $M M$ supplemented with Gly when many nitrogen sources such as Gly and Asp (data not shown) are added to MM separately. The disruption mutant M4018- $\Delta$ rim displayed significant increase in OTC production when cultivated on MM supplemented with $50 \mathrm{mM}$ glycine (Additional file 1: Figure S3). Thus, our experiments clearly demonstrated that Rim A1A2 negatively regulate OTC production in a medium-dependent manner. In contrast, in S. coelicolor, RimA1A2 homologue RapA1A2 regulates actinohrodin production positively when cultivated in R4C medium (Lu et al. 2007). Although OTC and ACT biosynthetically belong to the same group of metabolites, which belong to poliketides made of malonyl-CoA building blocks. Therefore, it is not surprising that these two metabolites are regulated in opposite direction by selected TCSs in these two streptomycetes. This most likely also depends on the composition of the medium used, considering that R4C medium consists of yeast extract and malt extract.

An SARP activator, OtcR, was identified later in oxy cluster of $S$. rimosus by Yin et al., which directly activate five promoters from oxy cluster. According to the qRTPCR analysis, the disruption of $\operatorname{rim} A 1$ could cause up regulation of the transcriptional level of otcR (Fig. 7). It is possible that RimA1A2 negatively regulates OTC production through the downregulation of otcR, thus acting as a global regulator. However, the RR RimA1-binding motif is not yet identified. Thus, at present, it is not possible to determine whether RimA1 directly interacts with the promoter regions of otcR by bioinformatics methods at this moment. This could be evaluated in the future work, whether RimA1 directly interacts with the promoter regions of five genes tested or other genes in the OTC cluster, which could potentially be detected by electrophoretic mobility shift assay (EMSA).
Pleiotropic effect of RimA1A2 in pigment formation and OTC production is not the only phenotypic change that we have observed in S. rimosus. We have observed other physiological and morphological properties of RimA1A2-inactivated mutant strain of $S$. rimosus.

\section{Impact of rimA1 disruption on OTC production when S. rimosus M4018 grown under different environmental stresses}

To evaluate the potential impact of rimA1 disruption on OTC production when $S$. rimosus M4018 $\Delta$ rim was cultivated under different environmental stresses, osmotic and oxidative stress were introduced into MM containing $50 \mathrm{mM}$ glycine. TCSs are the predominant form of signal transduction used by bacteria to respond against environmental stresses (Stock et al. 2000). Therefore, we set up experiments, where the $S$. rimosus growth rate and consequent secondary metabolite (OTC) production is evaluated when culture is exposed to $\mathrm{KCl}$ (osmotic pressure) or $\mathrm{H}_{2} \mathrm{O}_{2}$ (oxidative stress), respectively (Fig. 6). We have observed significant decrease in OTC production in the culture of mutant strain M4018 $\Delta$ rim (pIB-KA) (78\%), compared to M4018 (pIB-KA-rim) (21\%), when exposed to $\mathrm{KCl}$. Although we cannot bring any defined conclusions, the fact that WT strain with second copy of rimA1A2 expressed under strong constitutive promoter displays even lower response (only $21 \%$ reduction in OTC biosynthesis), it is reasonable to propose direct involvement of RimA1A2 into osmotic stress response.

Oxidative stress is common response to all aerobic organisms, but it is particularly pronounced in industrial biosynthetic processes for secondary metabolite production, where high intensity of oxygenation (aeration) is applied (Beites et al. 2011). A number of studies demonstrated that adaptive response of oxidative stress does not only have "defense role", but also has impact on the regulation of secondary metabolism. According to the literature, a number of transcriptional factors regulate expression of genes involved in oxidative stress response, which are regulated by different signaling pathways in the bacterial cell (Rao and Sureshkumar 2001; Wei et al. 2011; Hong et al. 2013; Montibus et al. 2015). The results on the oxidative stress response observed by treatment of the $S$. rimosus cultures by $\mathrm{H}_{2} \mathrm{O}_{2}$ were contrary to that of osmotic stress with $\mathrm{KCl}$ addition. The overexpression of rimA1A2 caused higher sensitivity of the culture to $\mathrm{H}_{2} \mathrm{O}_{2}$. Thus, it can be concluded that the function of RimA1A2 is rather to inhibit the process of oxidative stress response in the MM supplemented with glycine. However, considering that industrial strains with higher production of the target secondary metabolite can have oxidative stress response attenuated as demonstrated by Karnicar et al. (2016). The addition of $\mathrm{H}_{2} \mathrm{O}_{2}$ to 
the culture broth is an approach most often applied when evaluating the degree of oxidative stress. Thus, it can be hypothesized that RimA1A2 may play important role in the oxidative stress response. For the future experiments, where transcription profiles of different genes involved in the oxidative stress response in M4018 $\Delta$ rim strain or rimA1A2-overexpressed strains is compared, could give us more information on this hypothesis.

\section{Conclusions}

A TCS RimA1/A2 in S. rimosus was characterized in this work, displaying negative regulatory role in the biosynthesis of OTC in MM supplemented media with glycine as the sole nitrogen source. We have demonstrated that the transcription of a number of important genes in OTC biosynthesis was increased in the $\operatorname{rimA1-disrupted}$ mutant, thus demonstrating link between RimA1A2 and regulation of the genes from OTC cluster. We have also demonstrated the involvement of RimA1A2 in the regulation of OTC production under various environmental stresses conditions including increased osmolality and oxidative stress. These findings suggested that TCS RimA1A2 is among one of the global regulators to response different environmental stresses and regulate OTC production in a medium-dependent manner in $S$. rimosus $\mathrm{M} 4018$.

We have also highlighted the importance of cultivation conditions when studying the function of regulatory elements. Numerous genome-sequencing projects have recently revealed a huge number of "silent" gene clusters, which most often also contain different type of regulatory elements, present in almost every antibiotic-producing bacterium. These clusters are encoding potentially useful metabolites, which have not been identified, considering that they are not produced under standard laboratory conditions. Therefore, this work emphasizes the importance of cultivation conditions when studying the function of regulatory elements and production of secondary metabolites, which is often neglected.

This study also emphasizes the importance of medium composition and cultivation conditions applied in the scope of initial secondary metabolite screening and industrial strain improvement regimes. Moreover, the response of the industrial strains to mutagenesis and selection procedures greatly depends on the selection of the medium composition and cultivation conditions, this way directing isolation of strains with desired properties, often neglected at the industrial settings. It is, therefore, not that surprising that antibiotic-producing strains generally have such a large number of TCSs and complex regulatory network in general, this way ensuring high adaptation capacities of this microorganism to the changes in the environment.

\section{Additional file}

Additional file 1: Figure S1. Co-transcription analysis of the TCS genes rimA1/A2 of S. rimosus M4018. Figure S2. Morphological properties and pigmentation intensity of the parental strain M4018 and M4018 $\Delta$ rim on MM medium supplemented with different carbon sources (A: glucose, B: D-mannitol, C: lactose, D: arabinose, E: starch, F: maltose). Figure S3. Morphological properties and pigmentation intensity of M4018 and rimA1-disrupted strain tested on the MM supplemented with $50 \mathrm{mM}$ asparagine (A) or glycine (B).

\section{Abbreviations}

S. rimosus: Streptomyces rimosus; TCS: two-component system; ACT: actinorhodin; RR: response regulator; HK: histidine kinase; BTAD: bacterial transcriptional activation domain; MM: minimal medium; OTC: oxytetracycline; SARP: Streptomyces antibiotic regulatory protein.

\section{Authors' contributions}

$\mathrm{HN}$ and MG conceived and designed the study. HN performed experiments, contributed collection and assembly of data, data analysis and interpretation, and manuscript writing. ZX performed the computational analysis. ZX, AM, $M G, H K, J C$, and $Y Z$ revised the manuscript. All authors read and approved the final manuscript.

\section{Author details}

${ }^{1}$ State Key Laboratory of Bioreactor Engineering, East China University of Science and Technology, P.O. box 329\#, 130 Meilong Rd, Shanghai 200237, People's Republic of China. ${ }^{2}$ Shanghai Engineering Research Center of Food Microbiology, School of Medical Instrument and Food Engineering, University of Shanghai for Science and Technology, Shanghai 200093, China. ${ }^{3}$ Biotechnical Faculty, University of Ljubljana, Jamnikarjeva 101, 1000 Ljubljana, Slovenia.

\section{Acknowledgements}

Not applicable.

\section{Competing interests}

The authors declare that they have no competing interests.

Availability of data and materials

All data generated or analyzed during this study are included in this published article and its Additional files.

\section{Consent for publication}

Not applicable.

Ethics approval and concept to participate

Not applicable.

Funding

This research was supported by the Fundamental Research Funds for the Central Universities (No. 22221818014) to MG. This work as also supported by Slovenian Research Agency ARRS (Nos. J4-7637 and J4-8226) to HP.

\section{Publisher's Note}

Springer Nature remains neutral with regard to jurisdictional claims in published maps and institutional affiliations.

Received: 16 October 2018 Accepted: 7 January 2019

Published online: 21 January 2019 


\section{References}

Adamidis T, Riggle P, Champness W (1990) Mutations in a new Streptomyces coelicolor locus which globally block antibiotic biosynthesis but not sporulation. J Bacteriol 172:2962-2969

Ahn SJ, Qu MD, Roberts E, Burne RA, Rice KC (2012) Identification of the Streptococcus mutans LytST two-component regulon reveals its contribution to oxidative stress tolerance. BMC Microbiol 12:187

Bang IS, Kim BH, Foster JW, Park YK (2000) OmpR regulates the stationaryphase acid tolerance response of Salmonella enterica serovar typhimurium. J Bacteriol 182:2245-2252

Beites T, Pires SDS, Santos CL, Osório H, Moradas-Ferreira P, Mendes MV (2011) Crosstalk between ROS homeostasis and secondary metabolism in S. natalensis ATCC 27448: modulation of pimaricin production by intracellular ROS. PLOS ONE 6:e27472

Bentley SD, Chater KF, Cerdeno-Tarraga A, Challis GL, Thomson NR, James KD, Harris DE, Quail MA, Kieser H, Harper D (2002) Complete genome sequence of the model actinomycete Streptomyces coelicolor A3(2). Nature 417:141-147

Bierman M, Logan R, O'Brien K, Seno ET, Nagaraja Rao R, Schoner BE (1992) Plasmid cloning vectors for the conjugal transfer of DNA from Escherichia coli to Streptomyces spp. Gene 116:43-49

Bijlsma JJE, Groisman EA (2003) Making informed decisions: regulatory interactions between two-component systems. Trends Microbiol 11:359-366

Bishop A, Fielding S, Dyson P, Herron P (2003) Systematic insertional mutagenesis of a Streptomycete genome: a link between osmoadaptation and antibiotic production. Genome Res 14:893-900

Brian P, Riggle PJ, Santos RA, Champness WC (1996) Global negative regulation of Streptomyces coelicolor antibiotic synthesis mediated by an absAencoded putative signal transduction system. J Bacteriol 178:3221-3231

Bustin SA, Benes V, Garson JA, Hellemans J, Huggett J, Kubista M, Mueller R, Nolan T, Pfaffl MW, Shipley GL, Vandesompele J, Wittwer CT (2009) The MIQE guidelines: minimum information for publication of quantitative Real-Time PCR experiments. Clin Chem 55:611-622

Djoric D, Kristich CJ (2015) Oxidative stress enhances cephalosporin resistance of Enterococcus faecalis through activation of a two-component signaling system. Antimicrob Agents Chemother 59:159-169

Elsayed EA, Omar HG, El-Enshasy HA (2015) Development of fed-batch cultivation strategy for efficient oxytetracycline production by Streptomyces rimosus at semi-industrial scale. Braz Arch Biol Technol 58:676-685

Fabret C, Feher VA, Hoch JA (1999) Two-component signal transduction in Bacillus subtilis: how one organism sees its world. J Bacteriol 181:1975-1983

Finlay AC, Hobby GL, P'an SY, Regna PP, Routien JB, Seeley DB, Shull GM, Sobin BA, Solomons IA, Vinson JW, Kane JH (1950) Terramycin, a new antibiotic. Science 111:85

Finn RD, Coggill P, Eberhardt RY, Eddy SR, Mistry J, Mitchell AL, Potter SC, Punta M, Qureshi M, Sangrador-Vegas A, Salazar GA, Tate J, Bateman A (2016) The Pfam protein families database: towards a more sustainable future. Nucleic Acids Res 44:D279-D285

Hakenbeck R, Stock JB (1996) Analysis of two-component signal transduction systems involved in transcriptional regulation. Methods Enzymol 273:281-300

Hoch JA, Silhavy TJ (1995) Two-component signal transduction. ASM Press, Washington, D.C

Hong S, Roze LV, Linz JE (2013) Oxidative stress-related transcription factors in the regulation of secondary metabolism. Toxins 5:683-702

Hopwood DA, Bibb MJ, Chater KF, Kieser T, Bruton CJ, Kieser HM, Lydiate DJ, Smith CP, Ward JM, Schrempf H (1985) Genetic manipulation of Streptomyces: a laboratory manual. John Innes Foundation, Norwich

Hutchings MI, Hoskisson PA, Chandra G, Buttner MJ (2004) Sensing and responding to diverse extracellular signals? Analysis of the sensor kinases and response regulators of Streptomyces coelicolor A3(2). Microbiology 150:2795-2806

Karnicar K, Drobnak I, Petek M, Magdevska V, Horvat J, Vidmar R, Baebler S, Rotter A, Jamnik P, Fujs S, Turk B, Fonovic M, Gruden K, Kosec G, Petkovic $H$ (2016) Integrated omics approaches provide strategies for rapid erythromycin yield increase in Saccharopolyspora erythraea. Microb Cell Fact 15:1-17

Kieser T, Bibb MJ, Buttner MJ, Chater KF, Hopwood DA (2000) Practical streptomyces genetics. The John Innes Foundation, Norwich
Kim D, Forst S (2001) Genomic analysis of the histidine kinase family in bacteria and archaea. Microbiology 147:1197-1212

Kumar S, Stecher G, Tamura K (2016) MEGA7: molecular evolutionary genetics analysis version 7.0 for bigger datasets. Mol Biol Evol 33:1870-1874

Lesnik U, Gormand A, Magdevska V, Fujs S, Raspor P, Hunter I, Petkovic H (2009) Regulatory elements in tetracycline-encoding gene clusters: the otc $G$ gene positively regulates the production of oxytetracycline in Streptomyces rimosus. Food Technol Biotechnol 47:323-330

Livak KJ, Schmittgen TD (2001) Analysis of relative gene expression data using real-time quantitative PCR and the $2^{-\Delta \Delta C}$ method. Methods 25:402-408

Lu YH, Wang WH, Shu D, Zhang WW, Chen L, Qin ZJ, Yang S, Jiang WH (2007) Characterization of a novel two-component regulatory system involved in the regulation of both actinorhodin and a type I polyketide in Streptomyces coelicolor. Appl Microbiol Biotechnol 77:625-635

MacNeil DJ, Gewain KM, Ruby CL, Dezeny G, Gibbons PH, MacNeil T (1992) Analysis of Streptomyces avermitilis genes required for avermectin biosynthesis utilizing a novel integration vector. Gene 111:61-68

Mascher T, Helmann JD, Unden G (2006) Stimulus perception in bacterial signal-transducing histidine kinases. Microbiol Mol Biol Rev 70:910-938

McKenzie NL, Nodwell JR (2007) Phosphorylated AbsA2 negatively regulates antibiotic production in Streptomyces coelicolor through interactions with pathway-specific regulatory gene promoters. J Bacteriol 189:5284-5292

McMurry LM, Levy SB (1998) Revised sequence of OtrB (Tet347) tetracycline efflux protein from Streptomyces rimosus. Antimicrob Agents Chemother 42:3050

Montibus M, Pinson-Gadais L, Richard-Forget F, Barreau C, Ponts N (2015) Coupling of transcriptional response to oxidative stress and secondary metabolism regulation in filamentous fungi. Crit Rev Microbiol 41:295-308

Ogura M, Tanaka T (2002) Recent progress in Bacillus subtilis two-component regulation. Front Biosci 7:D1815-D1824

Ohnuki T, Katoh T, Imanaka T, Aiba S (1985) Molecular cloning of tetracycline resistance genes from Streptomyces rimosus in Streptomyces griseus and characterization of the cloned genes. J Bacteriol 161:1010-1016

Paget M, Leibovitz E, Buttner MJ (1999) A putative two-component signal transduction system regulates sigma(E), a sigma factor required for normal cell wall integrity in Streptomyces coelicolor A3(2). Mol Microbiol 33:97-107

Pethick FE, MacFadyen AC, Tang Z, Sangal V, Liu T, Chu J, Kosec G, Petkovic H, Guo M, Kirby R, Hoskisson PA, Herron PR, Hunter IS (2013) Draft genome sequence of the oxytetracycline-producing bacterium Streptomyces rimosus ATCC 10970. Genome Announc 1:1-2

Petkovic H, Cullum J, Hranueli D, Hunter IS, Peric-Concha N, Pigac J, Thamchaipenet A, Vujaklija D, Long PF (2006) Genetics of Streptomyces rimosus, the oxytetracycline producer. Microbiol Mol Biol Rev 70:704-728

Petkovic H, Lukezic T, Suskovic J (2017) Biosynthesis of oxytetracycline by Streptomyces rimosus: past, present and future directions in the development of tetracycline antibiotics. Food Technol Biotechnol 55:3-13

Pickens LB, Tang Y (2010) Oxytetracycline biosynthesis. J Biol Chem 285:27509-27515

Rao YM, Sureshkumar GK (2001) Improvement in bioreactor productivities using free radicals: $\mathrm{HOCl}$-induced overproduction of xanthan gum from Xanthomonas campestris and its mechanism. Biotechnol Bioeng 72:62-68

Rhodes PM, Hunter IS, Friend EJ, Warren M (1984) Recombinant DNA methods for the oxytetracycline producer Streptomyces rimosus. Biochem Soc T 12:586-587

Rodriguez H, Rico S, Diaz M, Santamaria RI (2013) Two-component systems in Streptomyces: key regulators of antibiotic complex pathways. Microb Cell Fact 12:1-10

Rodriguez-Garcia A, Barreiro C, Santos-Beneit F, Sola-Landa A, Martin JF (2007) Genome-wide transcriptomic and proteomic analysis of the primary response to phosphate limitation in Streptomyces coelicolor M145 and in a $\triangle$ phoP mutant. Proteomics 7:2410-2429

Rozas D, Gullon S, Mellado RP (2012) A novel two-component system involved in the transition to secondary metabolism in Streptomyces coelicolor. PLoS ONE 7:1-10

Ryding NJ, Anderson TB, Champness WC (2002) Regulation of the Streptomyces coelicolor calcium-dependent antibiotic by absA, encoding a clusterlinked two-component system. J Bacteriol 184:794-805

Sambrook J, Fritsch EF, Maniatis T (1989) Molecular cloning: a laboratory manual. Cold spring harbor laboratory press, New York 
Santos-Beneit F, Rodriguez-Garcia A, Sola-Landa A, Martin JF (2009) Cross-talk between two global regulators in Streptomyces: PhoP and AfsR interact in the control of afs S, pstS and phoRP transcription. Mol Microbiol 72:53-68

Shu D, Chen L, Wang WH, Yu ZY, Ren C, Zhang WW, Yang S, Lu YH, Jiang WH (2009) afsQ1-Q2-sigQ is a pleiotropic but conditionally required signal transduction system for both secondary metabolism and morphological development in Streptomyces coelicolor. Appl Microbiol Biotechnol 81:1149-1160

Singh N, Rai V, Tripathi C (2012) Production and optimization of oxytetracycline by a new isolate Streptomyces rimosus using response surface methodology. Med Chem Res 21:3140-3145

Sola-Landa A, Moura RS, Martin JF (2003) The two-component PhoR-PhoP system controls both primary metabolism and secondary metabolite biosynthesis in Streptomyces lividans. Proc Natl Acad Sci USA 100:6133-6138

Sola-Landa A, Rodriguez-Garcia A, Franco-Dominguez E, Martin JF (2005) Binding of PhoP to promoters of phosphate-regulated genes in Streptomyces coelicolor: identification of PHO boxes. Mol Microbiol 56:1373-1385

Sola-Landa A, Rodriguez-Garci A, Apel AK, Martin JF (2008) Target genes and structure of the direct repeats in the DNA-binding sequences of the response regulator PhoP in Streptomyces coelicolor. Nucleic Acids Res 36:1358-1368

Stock JB, Ninfa AJ, Stock AM (1989) Protein phosphorylation and regulation of adaptive responses in bacteria. Microbiol Rev 53:450-490

Stock AM, Robinson VL, Goudreau PN (2000) Two-component signal transduction. Annu Rev Biochem 69:183-215

Sugiura A, Hirokawa K, Nakashima K, Mizurto T (1994) Signal-sensing mechanisms of the putative osmosensor KdpD in Escherichia coli. Mol Microbiol 14:929-938

Taylor S, Wakem M, Dijkman G, Alsarraj M, Nguyen M (2010) A practical approach to RT-qPCR_-publishing data that conform to the MIQE guidelines. Methods 50:S1-S5
Wang JY, Yang SS (1995) Morphogenesis, biomass and oxytetracycline production of Streptomyces rimosus in submerged cultivation. Chin J Microbiol Immunol 28:21-31

Wang R, Mast Y, Wang J, Zhang WW, Zhao GP, Wohlleben W, Lu YH, Jiang WH (2013) Identification of two-component system AfsQ1/Q2 regulon and its cross-regulation with GInR in Streptomyces coelicolor. Mol Microbiol 87:30-48

Wei Z, Bai L, Deng Z, Zhong J (2011) Enhanced production of validamycin A by $\mathrm{H}_{2} \mathrm{O}_{2}$-induced reactive oxygen species in fermentation of Streptomyces hygroscopicus 5008. Bioresour Technol 102:1783-1787

Wietzorrek A, Bibb M (1997) A novel family of proteins that regulates antibiotic production in streptomycetes appears to contain an OmpR-like DNAbinding fold. Mol Microbiol 25:1181-1184

Wilkinson CJ, Hughes-Thomas ZA, Martin CJ, Bohm I, Mironenko T, Deacon M, Wheatcroft M, Wirtz G, Staunton J, Leadlay PF (2002) Increasing the efficiency of heterologous promoters in actinomycetes. J Mol Microbiol Biotechnol 4:417-426

Wood JM (1999) Osmosensing by bacteria: signals and membrane-based sensors. Microbiol Mol Biol Rev 63:230-262

Yin SL, Wang WS, Wang XF, Zhu YX, Jia XL, Li SS, Yuan F, Zhang YX, Yang KQ (2015) Identification of a cluster-situated activator of oxytetracycline biosynthesis and manipulation of its expression for improved oxytetracycline production in Streptomyces rimosus. Microb Cell Fact 14:1-12

Yu L, Yan X, Wang L, Chu J, Zhuang Y, Zhang S, Guo M (2012a) Molecular cloning and functional characterization of an ATP-binding cassette transporter OtrC from Streptomyces rimosus. BMC Biotechnol 12:52

Yu ZY, Zhu H, Dang FJ, Zhang WW, Qin ZJ, Yang S, Tan HR, Lu YH, Jiang WH (2012b) Differential regulation of antibiotic biosynthesis by DraR-K, a novel two-component system in Streptomyces coelicolor. Mol Microbiol $85: 535-556$

\section{Submit your manuscript to a SpringerOpen ${ }^{\odot}$ journal and benefit from:}

- Convenient online submission

- Rigorous peer review

- Open access: articles freely available online

- High visibility within the field

Retaining the copyright to your article

Submit your next manuscript at $\boldsymbol{\nabla}$ springeropen.com 